\title{
Immunohistochemistry is highly sensitive and specific for detection of BRAF V600E mutation in pleomorphic xanthoastrocytoma
}

\author{
Cristiane M Ida ${ }^{1}$, Julie A Vrana ${ }^{1}$, Fausto J Rodriguez ${ }^{4}$, Mark E Jentoft ${ }^{1}$, Alissa A Caron ${ }^{2}$, Sarah M Jenkins ${ }^{3}$ and

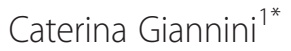

\begin{abstract}
Background: High frequencies of the BRAF V600E mutation have been reported in pleomorphic xanthoastrocytoma (PXA). Recently, a BRAF V600E mutation-specific antibody has been developed and validated. We evaluated the immunohistochemical (IHC) detection of BRAF V600E mutation in PXA by comparing to gold standard molecular analysis and investigating the interobserver variability of the IHC scoring. We performed BRAF V600E IHC in 46 cases, of which 37 (80\%) cases had sufficient tumor tissue for molecular analysis. IHC detection was performed using monoclonal mouse antibody VE1 (Spring Bioscience). IHC slides were scored independently by four reviewers blind to molecular data, including a primary (gold standard) and three additional reviewers. BRAF V600E mutation status was assessed by allele-specific polymerase chain reaction (PCR) with fragment analysis.

Results: All 46 cases showed interpretable BRAF V600E IHC results: 27 (59\%) were positive (strong cytoplasmic staining), 19 (41\%) were negative (6 of these cases with focal/diffuse weak cytoplasmic staining, interpreted as nonspecific by the primary reviewer). By molecular analysis, all 37 cases that could be tested had evaluable results: 22 (59\%) cases were positive for BRAF V600E mutation and were scored as "IHC-positive", and 15 (41\%) were negative (including 11 cases scored as "IHC-negative" and 4 cases scored as negative with minimal nonspecific staining). IHC detection of BRAF V600E mutant protein was congruent in all 37 cases that were successfully evaluated by molecular testing (sensitivity and specificity of 100\%). Agreement for $\mathrm{IHC}$ scoring among the 4 reviewers was almost perfect (kappa 0.92) when cases were scored as "positive/negative" and substantial (kappa 0.78) when minimal nonspecific staining was taken into account.

Conclusions: We conclude that detection of BRAF V600E mutation by immunohistochemistry is highly sensitive and specific. BRAF V600E IHC interpretation is usually straightforward, but awareness of possible nonspecific staining is necessary and training is recommended. It is a practical rapid method that may avoid the need of labor-intensive molecular testing and may be most valuable in small biopsies unsuitable for molecular analysis.
\end{abstract}

Keywords: Pleomorphic xanthoastrocytoma, BRAF V600E, Immunohistochemistry

\section{Background}

BRAF, a member of the RAF family including ARAF, BRAF and RAF1, is a serine/threonine protein kinase encoded by $B R A F$ gene on chromosome $7 \mathrm{q} 34$ that activates the MAP kinase/ERK-signaling pathway mediating cellular responses to growth signals. It is the family member that is

\footnotetext{
*Correspondence: giannini.caterina@mayo.edu

'Departments of Laboratory Medicine and Pathology, Mayo Clinic, Rochester, MN, USA

Full list of author information is available at the end of the article
}

most easily activated by RAS, and the one with highest kinase activity [1-3]. Frequent somatic mutational activation of $B R A F$ has been observed in human cancers, including melanomas, gliomas, colorectal cancers, lung cancers and others [4]. Among primary central nervous system (CNS) neoplasms [5-9], activation of the MAP kinase/ ERK-signaling pathway appears to play an important role in the pathogenesis of a subset of glial/glioneuronal tumors, in particular, pilocytic astrocytoma (PA) [8-13], PXA [14], ganglioglioma (GG) $[9,15]$, and dysembryoplastic

\section{Biomed Central}


neuroepithelial tumor (DNET) [16]. BRAF activation in PA primarily results from tandem duplications at $7 \mathrm{q} 34$ with subsequent fusion between the 5 ' end of a gene of unknown function, KIAA1549, and the 3' end of BRAF; while in PXA, GG and DNET, constitutive $B R A F$ activation results from heterozygous missense mutation at codon 600 (V600E). $B R A F$ V600E mutation is characterized by exchange of $\mathrm{T}$ to $\mathrm{A}$ at base position c.1799 (c. $1799 \mathrm{~T}>\mathrm{A}$ ), which results in substitution of glutamic acid by valine at residue 600 (p. Val600Glut). Less frequent activating BRAF mutations (e.g. V600K, V600D, V600M) have been observed in malignant melanoma [17-20] and other non-CNS tumors $[4,21]$ but only rarely have been identified in primary CNS tumors [6].

The highest frequencies of $B R A F$ V600E mutation in primary CNS neoplasms have been reported in PXA (up to 60-65\%) [8,9,14,22], a WHO grade II tumor [23], with $30 \%$ recurrence and $80 \%$ overall survival rates at five years following primary resection. Histologically, PXA is characterized by marked cellular pleomorphism, nuclear atypia, and a variable number of bizarre, multinucleate giant cells ("classic PXA"), and occasionally shows increased mitotic activity and/or necrosis ("PXA with anaplastic features") [23,24]. The main morphological differential diagnosis of PXA includes other pleomorphic and often more aggressive tumors such as glioblastoma (GBM)/giant cell or epithelioid GBM, a World Health Organization (WHO) grade IV tumor [23]. Such critical clinical distinction with important prognostic and clinical implications may be morphologically challenging. Of note, $B R A F$ V600E mutation has been found in low frequency among GBM/giant cell GBM (approximately 5-10\%) $[5,7,14]$, but in up to $54 \%$ among epithelioid GBM [25]. Therefore, BRAF V600E mutation assessment may be a potentially useful marker in the differential diagnosis of GBM/giant cell GBM vs. "PXA with anaplastic features" and in identifying $B R A F$ V600E mutant astrocytic tumors suitable for targeted therapy.

Recently, a $B R A F$ V600E mutation-specific monoclonal antibody has been developed [26] and validated as a reliable test among tumors that frequently harbor the BRAF V600E mutation, including primary and metastatic melanoma $[18,20,27,28]$, papillary thyroid carcinoma $[29,30]$, hairy cell leukemia [31], ovarian serous borderline tumors [32], primary lung adenocarcinomas [33], as well as in a large series of brain metastases and corresponding non-CNS primary tumors [34].

Herein, we evaluated the IHC detection of BRAF V600E mutant protein in PXA by comparing to BRAF V600E mutation detection by molecular analysis, and investigated the interobserver variability of the IHC scoring. Detection of $B R A F$ V600E mutation in PXA by immunohistochemistry was highly sensitive and specific, and showed a substantial/ almost perfect interobserver agreement.

\section{Results}

$B R A F$ V600E mutation immunohistochemical and molecular analyses

All 46 (100\%) cases with available tissue were evaluable by immunohistochemistry for BRAF V600E mutant protein. Cases were scored as positive if non-ambiguous tumor cell cytoplasmic staining was identified ("IHC-positive"). BRAF V600E immunoreactivity in most cases was characterized by intense cytoplasmic stain, with a somewhat granular appearance, likely due to the visualization/amplification set used and with variable distribution. In some cases, all tumor cells stained; while in others, the stain was patchy in the tissue sections. Isolated tumor cells infiltrating parenchyma were also visualized by the immunostain. According to the primary reviewer, 27 (59\%) cases were "IHC-positive," and 19 (41\%) cases were "IHC-negative," including 6 cases "IHC-negative/nonspecific." Examples of immunostaining pattern are shown in Figures 1 and 2 . Immunostaining varied in intensity from strong (Figure 1A) to weak (Figure 1B), as well as in distribution, with cases showing isolated infiltrating tumor cells (Figure 1C). Among cases considered negative ("IHC-negative"), although the majority of cases did not have any degree of immunostaining (Figure 2A), a few showed very focal and/or weak tumor cell cytoplasmic staining as shown in Figures $2 \mathrm{~B}$ and $2 \mathrm{C}$. This focal/weak immunostaining was interpreted as nonspecific ("IHC-negative/nonspecific").

Among the 37 (of 46, 80\%) cases with sufficient tumor tissue for molecular analysis: 22 (of 37, 59\%) were positive for BRAF V600E mutation, including 16 classic PXA and 6 PXA with anaplastic features, and 15 (41\%) cases were negative for BRAF V600E mutation, including 7 classic PXA and 8 PXA with anaplastic features. All 22 cases positive for BRAF V600E mutation (Figure 1D; Additional file 1: Figure S1 for complete electropherogram panel) had been scored as "IHC-positive." Of the 15 cases negative for $B R A F$ V600E mutation (Figure 2D; Additional file 2: Figure S2 for complete electropherogram panel), 11 had been scored as "IHC-negative" and 4 as "IHC-negative/ nonspecific" cases (Table 1). Therefore, there was complete agreement between $B R A F$ V600E mutation molecular analysis and IHC detection of BRAF V600E mutant protein in all 37 cases, with sensitivity of $100 \%$ (confidence interval 85.1-100) and specificity of $100 \%$ (79.6-100) according to the primary reviewer.

\section{Interobserver variability of BRAF V600E immunohistochemical analysis}

Most (26) cases scored as "IHC-positive" by the primary reviewer were also interpreted as "IHC-positive" by the other three reviewers (Figure 3: Table 2 ), with the exception of one "IHC negative/nonspecific" interpretation by a single reviewer. However, reviewers three and four also scored as positive one additional case among those 


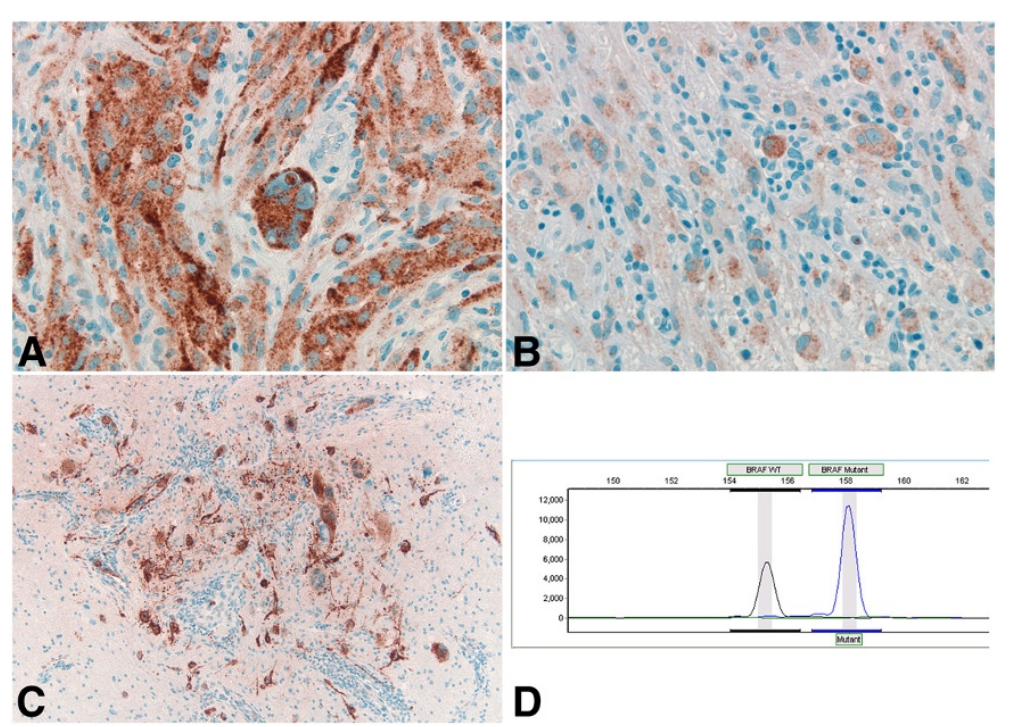

Figure 1 BRAF V600E IHC positive PXA cases molecularly confirmed as BRAF V600E mutant tumors: case 17- strong granular cytoplasmic immunostaining of a characteristic pleomorphic multinucleated giant tumor cell (A), case 3- weak granular cytoplasmic immunostaining of pleomorphic and spindle tumor cells (B), case 10- strong granular cytoplasmic immunostaining of a cluster of isolated tumor cells (C), 400X; and case 14- mutant BRAF V600E peak in addition to the wild-type BRAF peak, consistent with presence of BRAF V600E mutation (D).

scored as "IHC-negative/nonspecific" by the primary reviewer (Figure 3: Table 2 ). All 13 cases scored as "IHC-negative" by the primary reviewer were also scored as "IHC-negative" by reviewers two and three, while reviewer four scored two of them as "IHC-negative/ nonspecific." The results of the review of the remaining six cases interpreted as "IHC-negative/nonspecific" by the primary reviewer as well as the results of the $B R A F$ V600E mutation molecular analysis are detailed in Figure 3: Table 2. In summary: two cases were scored as "IHC-negative" by the other three observers; two cases were also scored as "IHC-negative/nonspecific"

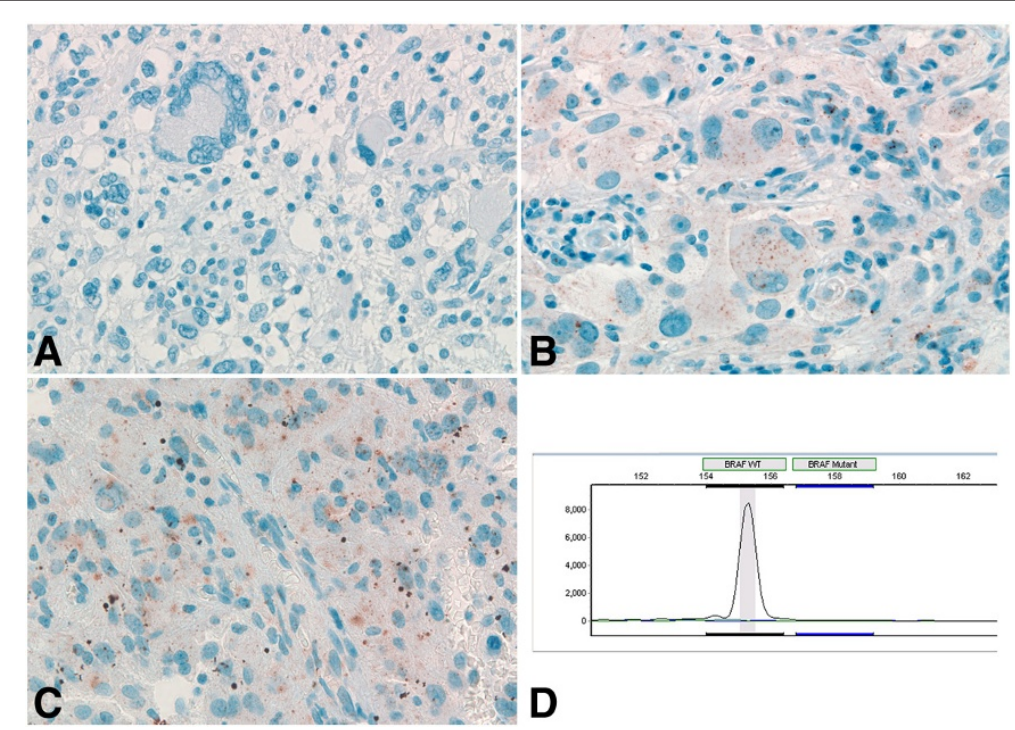

Figure 2 BRAF V600E IHC negative \& "negative/non-specific" PXA cases molecularly confirmed as BRAF V600E non-mutant tumors: case 38- BRAF V600E IHC negative case characterized by complete lack of tumor cell immunostaining (A), case 20- BRAF V600E IHC "negative/non-specific" case showing focal weak granular cytoplasmic immunostaining of tumor cells (B), case 47- BRAF V600E IHC "negative/non-specific" case with focal weak granular cytoplasmic immunostaining of tumor cells in addition to small extracellular immunostaining precipitate background (C); 400X; and case 20-BRAF wild-type peak without mutant BRAF V600E peak, consistent with the absence of BRAF V600E mutation and in keeping with the negative BRAFV600E IHC (D). 
Table 1 BRAF V600E mutation and BRAF V600E IHC (Primary reviewer) comparison $(n=37)$

\begin{tabular}{lccc}
\hline BRAF V600E Mutation & \multicolumn{3}{c}{ BRAF V600E IHC } \\
\cline { 2 - 4 } & Positive & Negative & Negative/Nonspecific \\
\hline Mutant & 22 & 0 & 0 \\
Non-mutant & 0 & 11 & 4 \\
\hline
\end{tabular}

by one observer and as "IHC-negative" by the other two observers; one case was scored as "IHC-negative" by two observers and as "IHC-positive" by one observer; one case was also scored as "IHC-negative/nonspecific" by one observer and as "IHC-positive" by the other two observers.

Agreement for IHC scoring was almost perfect (kappa 0.92) when cases were scored as "positive/negative" ("IHC-positive" vs. "IHC-negative") and substantial (kappa 0.78) when "negative/nonspecific" staining was taken into account ("IHC-positive" vs. "IHC-negative" vs. "IHC-negative-nonspecific").

\section{Discussion}

In keeping with the majority of previous studies that also evaluated the immunohistochemical detection of the BRAF V600E mutation in comparison to gold standard molecular testing in a variety of tumor types [16,18,20,26-34], we concluded that immunohistochemistry is an accurate detection method for the BRAF V600E mutation in PXA. In our study, IHC results were comparable to the gold standard molecular testing for identification of $B R A F$ V600E mutation and resulted in high sensitivity and specificity. In contrast to the labor-intensive detection of BRAF V600E mutation by molecular testing, identification of BRAF V600E mutant protein by immunohistochemistry is a rapid method that may be quickly implemented in diagnostic pathology practice since it is a widespread technique available in most academic centers as well as in non-academic pathology practices. However, validation of the BRAF V600E mutation IHC specificity by comparative molecular analysis should be performed for each tumor entity before routine diagnostic implementation, since it has been recently reported that in pituitary adenomas, the BRAF V600E mutation-specific VE1 immunostaining is not associated with presence of $B R A F \mathrm{~V} 600 \mathrm{E}$ mutation [35].

In surgical neuropathology, BRAF V600E mutationspecific immunohistochemistry has a potential clinically relevant role. IHC detection of BRAF V600E mutant protein is an accurate and reliable alternative method that may be diagnostically useful when dealing with morphologically challenging pleomorphic astrocytic tumors in which the differential diagnoses include PXA and GBM/ giant cell GBM. In addition, BRAF V600E mutation has also been identified to a lesser extent in epithelioid GBM[25], ganglioglioma and pilocytic astrocytoma, predominantly in extra-cerebellar location [9], expanding the diagnostic repertoire use of BRAF V600E mutationspecific IHC testing among primary CNS tumors and potentially the identification of tumors suitable for $B R A F$ V600E mutation-targeted therapy.

Of note, low tumor cell content may prevent identification of the BRAF V600E mutation by molecular analysis and result in false-negative results, but this limitation has been shown to be overcome by the use of BRAF V600E monoclonal antibody $[18,29,30,32,34]$. Thus, small biopsies that would be deemed unsuitable for molecular testing may be successfully evaluated by immunohistochemistry. This is particularly important in surgical neuropathology, in which biopsy samples of small size and/or with low tumor cell

\begin{tabular}{|c|c|c|c|c|c|}
\hline \multicolumn{4}{|c|}{ Reviewer } & \multirow[b]{2}{*}{ BRAF V600E } & \multirow[b]{2}{*}{ Samples } \\
\hline Primary & Two & Three & Four & & \\
\hline \multicolumn{4}{|c|}{ Positive } & Mutant & 22 \\
\hline \multicolumn{4}{|c|}{ Positive } & ND & 4 \\
\hline Positive & Neg/NS & \multicolumn{2}{|c|}{ Positive } & ND & 1 \\
\hline \multicolumn{4}{|c|}{ Negative } & Non-mutant & 9 \\
\hline \multicolumn{4}{|c|}{ Negative } & $N D$ & 2 \\
\hline \multicolumn{3}{|c|}{ Negative } & Neg/NS & Non-mutant & 2 \\
\hline Neg/NS & \multicolumn{3}{|c|}{ Negative } & Non-mutant & 2 \\
\hline Neg/NS & \multicolumn{2}{|c|}{ Negative } & Neg/NS & Non-mutant & 2 \\
\hline Neg/NS & \multicolumn{2}{|c|}{ Negative } & Positive & ND & 1 \\
\hline Neg/NS & \multicolumn{2}{|c|}{ Positive } & Neg/NS & ND & 1 \\
\hline
\end{tabular}

Figure 3: Table 2 BRAF V600E IHC Interobserver variability $(n=46) . ~ N e g / N S=$ negative/non specific. ND = not done. 
content are not infrequent. In addition, BRAF V600E IHC analysis allows identification of tumor cells at the single cell level, which would not only aid in the diagnosis of biopsies with very low tumor cell content but would also allow localization of the mutation at a cellular type level, as recently highlighted by the demonstration of neuronal tumor cells as the predominant tumor cell population harboring the BRAF V600E mutation in gangliogliomas [15].

In our study, BRAF V600E IHC interpretation was usually straightforward with high (at least substantial) interobserver agreement. The presence of rare cases with nonspecific staining is a potential pitfall, which could lead to rare false positive results. Similarly, others have also reported negative cases with nonspecific background staining $[15,32,34]$. In ambiguous cases, sequential molecular confirmatory testing is advocated [20,27]. We recommend that pathologists not only be aware of the possibility of nonspecific staining but also train during validation of the IHC testing to correctly identify such cases and avoid misinterpretation of nonspecific staining as positive staining.

\section{Conclusions}

Detection of BRAF V600E mutation in PXA by immunohistochemistry is highly sensitive and specific with substantial/almost perfect interobserver agreement. BRAF V600E IHC interpretation is usually straightforward, but awareness of possible nonspecific staining is necessary, and training on the IHC stain interpretation is recommended. It is a practical rapid method that may avoid the need of labor-intensive molecular testing and may be extremely valuable in biopsies unsuitable for molecular analysis due to either small size or low tumor cell content.

\section{Methods}

\section{Case selection}

All studies were conducted according to Mayo Institutional Review Board-approved protocols. This study was approved by the Mayo IRB as a minimal risk study with waiver of consent.

According to the Minnesota law, the Minnesota research authorization status was reviewed and only patients whose Research Status was "yes" were included in the study.

Fifty pleomorphic xanthoastrocytoma (PXA) cases upon first surgical resection were selected from the files from Mayo Clinic $(\mathrm{n}=32)$ and Johns Hopkins University $(\mathrm{n}=18)$ from 1965 to 2011. All existing diagnostic slides were retrieved and reviewed by at least two of the authors (C.G. and C.M.I.), and the diagnosis of PXA was confirmed according to previously described criteria [23]. Tumors showed a relatively solid growth pattern and were composed of a combination of spindle-shaped, xanthic and pleomorphic, multinucleated giant astrocytes, associated with both pale and bright eosinophilic granular bodies. They included both classic PXA ( $\leq 5$ mitotic figures per 10 high power fields) and PXA with anaplastic features (including $>5$ mitotic figures per 10 high power fields and/ or necrosis). All 50 cases were classified as either classic PXA $(\mathrm{n}=34)$ or PXA with anaplastic features $(\mathrm{n}=16)$ and were included in the study. Of these, 46 cases $(92 \%)$ had available tissue for IHC and/or molecular analysis.

\section{BRAF V600E immunohistochemical analysis}

Four-micron freshly cut sections ( $<2$ weeks) of formalinfixed, paraffin-embedded (FFPE) tissue of 46 (of 50) cases were dried and melting at $62^{\circ} \mathrm{C}$ oven for 20 minutes. Subsequently, they were stained with mouse monoclonal BRAF V600E antibody (1/100 titer; clone VE1) and raised against a synthetic peptide corresponding to amino acids 596-606 (GLATEKSRWSG) of mutant BRAF (Spring Bioscience) with slight modifications to the manufacturer's protocol. Briefly, staining was performed on the Ventana BenchMark XT (Ventana Medical Systems Inc.). The staining protocol included online deparaffinization, HIER (Heat Induced Epitope Retrieval) with Ventana Cell Conditioning 1 for 32 minutes and primary antibody incubation for 32 minutes at $37^{\circ} \mathrm{C}$. Antigen-antibody reactions were visualized using Ventana OptiViewTM Amplification kit, followed by Ventana OptiViewTM Universal DAB Detection Kit (Optiview HQ Linker 8 min, Optiview HRP Multimer 8 min, Optiview Amplifier H2O2/ Amplifier $4 \mathrm{~min}$, Optiview Amplifier Multimer $4 \mathrm{~min}$, Optiview H2O2/DAB 8 min, Optiview Copper $4 \mathrm{~min}$ ). Counterstaining was obtained online using Ventana Hematoxylin II for 8 minutes followed by bluing reagent for 4 minutes. Finally, all slides are removed from the stainer, dehydrated, and coverslipped for microscopic examination. Positive control included a known BRAF V600E mutant skin malignant melanoma. Cases were scored as positive ("IHC-positive"), negative ("IHC-negative"), and "negative-nonspecific" ("IHC negative/nonspecific"). Only tumor cells showing non-ambiguous cytoplasmic staining for BRAF V600E immunostain were scored as positive ("IHC positive"). Faint, weak granular stain was noted in a few cases and considered nonspecific. These cases were scored as "IHC negative/nonspecific" for tracking purposes. IHC slides were scored first by a primary reviewer and later by three additional independent reviewers, all four blind to the molecular results. The primary reviewer (CG) was the one most intimately involved with the development of the immunostain, who had gained most experience through the process, and was most familiar with variations in stain intensity and distribution not only among PXA cases but also with other positive and negative controls. Because of the level of expertise with reading the BRAF V600E immunostain and of the sequence of events, the primary reviewer was used as the "gold standard" to which the results of the BRAF V600E mutation molecular analysis were compared (see below). The four reviewers were 
compared to each other in regards to the scoring of BRAF V600E immunohistochemistry.

\section{BRAF V600E mutation status by allele-specific PCR with fragment analysis}

After review of hematoxylin and eosin-stained slides, of all the 50 cases, $37(74 \%)$ cases had sufficient $(\geq 20 \%)$ viable tumor for BRAF V600E mutation molecular analysis. DNA was extracted from 5-micron sections of FFPE tissue (four to eight slides per case) using the QIAamp DNA FFPE Tissue Kit (Qiagen) according to the manufacturer's instructions with few modifications (samples were lysed overnight; $10 \mathrm{uL}$ of additional proteinase $\mathrm{K}$ was used on the following day; the tissue was allowed to complete lysis for additional one to two hours). DNA was quantified using a Qubit fluorometer and Qubit Quant-iT dsDNA BR Assay Kit (Invitrogen). Testing for the BRAF V600E mutation was performed following clinically validated protocols. $B R A F$ allele-specific fluorescent PCR was performed with primers specifically designed to detect the base mutant and wild type base at position c.1799, and fragment analysis was completed on the ABI 3730 DNA Analyzer (Applied Biosystems). The primers were differentially labeled and had a size variance in addition to the different fluorophore. Wild-type peak was approximately 155.2 base pairs, and the mutant peak was approximately 158 base pairs (predetermined bins were set at $+/-1.2$ base pairs from the expected size, i.e. $155.2+/-1.2 \mathrm{bp}$ and $158+/-1.2 \mathrm{bp})$.

\section{Statistical analysis}

Sensitivity and specificity along with 95\% confidence intervals (score method) were calculated for BRAF V600E $\mathrm{IHC}$ analysis based on the reading of the primary reviewer compared to the BRAF V600E mutation molecular analysis result considered the "gold standard".

To evaluate the degree of agreement for IHC interpretation among the four observers, overall kappa for $>2$ reviewers were calculated. Kappa values may vary from 0 to 1.0. Values of 0.4 to 0.6 are considered evidence of "moderate" agreement, >0.6-0.8 of "substantial," and >0.8-1 of "almost perfect" agreement according to Landis and Koch [36]. Kappa was calculated based on the IHC scoring results in two scenarios: when cases were scored as "positive/negative" and when negative/nonspecific staining was also taken into account [36-39]. All analyses were performed using SAS version 9 (Cary, NC).

\section{Additional files}

Additional file 1: Figure S1. Fragment analysis electropherograms of a BRAF V600E mutant case (case 14): mutant BRAF V600E peak in addition to the wild- type BRAF peak, consistent with presence of BRAF V600E mutation (A); positive control- mutant BRAF V600E peak in addition to the wild-type BRAF peak, consistent with presence of BRAF V600E mutation (B); negative control- BRAF wild-type peak without mutant BRAF V600E peak, consistent absence of BRAF V600E mutation (C); Blank/No DNA- Absence of distinct peaks, indicating no sample contamination (D).

Additional file 2: Figure S2. Fragment analysis electropherograms of a BRAF V600E non-mutant case (case 20): BRAF wild-type peak without mutant BRAF V600E peak, consistent with absence of BRAF V600E mutation (A); positive control- mutant BRAF V600E peak in addition to the wild-type BRAF peak, consistent with presence of BRAF V600E mutation (B); negative control- BRAF wild-type peak without mutant BRAF V600E peak, consistent absence of BRAF V600E mutation (C); Blank/No DNA- Absence of distinct peaks, indicating no sample contamination (D).

\section{Abbreviations}

PXA: Pleomorphic xanthoastrocytoma; IHC: Immunohistochemical; CNS: Central nervous system; PA: Pilocytic astrocytoma; GG: Ganglioglioma; DNET: Dysembryoplastic neuroepithelial tumor; WHO: World Health Organization; GBM: Glioblastoma; HIER: Heat-Induced Epitope Retrieval; PCR: Polymerase chain reaction; BP: Base pairs.

\section{Competing interests}

The authors declare that they have no competing interests.

\section{Authors' contributions}

Cristiane M. Ida, MD, planned project, reviewed cases, wrote manuscript. Julie A. Vrana performed BRAF V600E immunostaining, reviewed manuscript.

Fausto J. Rodriguez, MD, provided and reviewed part of the cases, reviewed manuscript. Mark E. Jentoft, MD, reviewed immunohistochemical stains, reviewed manuscript. Alissa A. Caron performed DNA extraction for BRAF V600E mutation molecular analysis, reviewed manuscript. Sarah M. Jenkins performed statistical analysis, reviewed manuscript. Caterina Giannini, MD, planned project, reviewed cases, prepared figures, wrote manuscript. All authors read and approved the final manuscript.

\section{Acknowledgements}

The authors would like to thank Dr. Kandelaria A. Rumilla for her help in preparing the photographs of the BRAF V600E mutation molecular analysis.

\section{Author details}

${ }^{1}$ Departments of Laboratory Medicine and Pathology, Mayo Clinic, Rochester, MN, USA. ${ }^{2}$ Experimental Pathology, Mayo Clinic, Rochester, MN, USA. ${ }^{3}$ Biomedical Statistics and Informatics, Mayo Clinic, Rochester, MN, USA. ${ }^{4}$ Pathology, Johns Hopkins University, Baltimore, MD, USA.

Received: 17 April 2013 Accepted: 7 May 2013

Published: 30 May 2013

\section{References}

1. Emuss V, Garnett M, Mason C, Marais R: Mutations of C-RAF are rare in human cancer because C-RAF has a low basal kinase activity compared with B-RAF. Cancer Res 2005, 65:9719-9726.

2. Niault TS, Baccarini M: Targets of Raf in tumorigenesis. Carcinogenesis 2010, 31:1165-1174.

3. Wellbrock C, Karasarides M, Marais R: The RAF proteins take centre stage. Nat Rev Mol Cell Biol 2004, 5:875-885.

4. Davies H, Bignell GR, Cox C, Stephens P, Edkins S, Clegg S, Teague J, Woffendin H, Garnett MJ, Bottomley W, et al: Mutations of the BRAF gene in human cancer. Nature 2002, 417:949-954.

5. Basto D, Trovisco V, Lopes JM, Martins A, Pardal F, Soares P, Reis RM: Mutation analysis of B-RAF gene in human gliomas. Acta Neuropathol 2005, 109:207-210.

6. Jeuken J, van den Broecke C, Gijsen S, Boots-Sprenger S, Wesseling P: RAS/RAF pathway activation in gliomas: the result of copy number gains rather than activating mutations. Acta Neuropathol 2007, 114:121-133.

7. Knobbe CB, Reifenberger J, Reifenberger G: Mutation analysis of the Ras pathway genes NRAS, HRAS, KRAS and BRAF in glioblastomas. Acta Neuropathol 2004, 108:467-470.

8. Myung JK, Cho H, Park CK, Kim SK, Lee SH, Park SH: Analysis of the BRAF (V600E) Mutation in Central Nervous System Tumors. Trans/ Oncol 2012, 5:430-436. 
9. Schindler G, Capper D, Meyer J, Janzarik W, Omran H, Herold-Mende C, Schmieder K, Wesseling P, Mawrin C, Hasselblatt M, et al: Analysis of BRAF V600E mutation in 1,320 nervous system tumors reveals high mutation frequencies in pleomorphic xanthoastrocytoma, ganglioglioma and extra-cerebellar pilocytic astrocytoma. Acta Neuropathol 2011, 121:397-405.

10. Dougherty MJ, Santi M, Brose MS, Ma C, Resnick AC, Sievert AJ, Storm PB, Biegel JA: Activating mutations in BRAF characterize a spectrum of pediatric low-grade gliomas. Neuro Oncol 2010, 12:621-630.

11. Forshew T, Tatevossian RG, Lawson AR, Ma J, Neale G, Ogunkolade BW, Jones TA, Aarum J, Dalton J, Bailey S, et al: Activation of the ERK/MAPK pathway: a signature genetic defect in posterior fossa pilocytic astrocytomas. J Pathol 2009, 218:172-181.

12. Lin A, Rodriguez FJ, Karajannis MA, Williams SC, Legault G, Zagzag D, Burger PC, Allen JC, Eberhart CG, Bar EE: BRAF alterations in primary glial and glioneuronal neoplasms of the central nervous system with identification of 2 novel KIAA1549:BRAF fusion variants. J Neuropathol Exp Neurol 2012, 71:66-72.

13. Rodriguez FJ, Ligon AH, Horkayne-Szakaly I, Rushing EJ, Ligon KL, Vena N, Garcia DI, Cameron JD, Eberhart CG: BRAF duplications and MAPK pathway activation are frequent in gliomas of the optic nerve proper. J Neuropathol Exp Neurol 2012, 71:789-794.

14. Dias-Santagata D, Lam Q, Vernovsky K, Vena N, Lennerz JK, Borger DR, Batchelor TT, Ligon $\mathrm{KL}$, lafrate $\mathrm{AJ}$, Ligon $\mathrm{AH}$, et al: BRAF V600E mutations are common in pleomorphic xanthoastrocytoma: diagnostic and therapeutic implications. PLoS One 2011, 6:e17948.

15. Koelsche C, Wöhrer A, Jeibmann A, Schittenhelm J, Schindler G, Preusser M, Lasitschka F, Deimling A, Capper D: Mutant BRAF V600E protein in ganglioglioma is predominantly expressed by neuronal tumor cells. Acta Neuropathol 2013, 125:891-900.

16. Chappe C, Padovani L, Scavarda D, Forest F, Nanni-Metellus I, Loundou A, Mercurio S, Fina F, Lena G, Colin C, Figarella-Branger D: Dysembryoplastic Neuroepithelial Tumors Share with Pleomorphic Xanthoastrocytomas and Gangliogliomas BRAF Mutation and Expression. Brain Pathol 2013. Epub ahead of print.

17. Amanuel B, Grieu F, Kular J, Millward M, lacopetta B: Incidence of BRAF p. Val600Glu and p.Val600Lys mutations in a consecutive series of 183 metastatic melanoma patients from a high incidence region. Pathology 2012, 44:357-359.

18. Long GV, Wilmott JS, Capper D, Preusser M, Zhang YE, Thompson JF, Kefford RF, von Deimling A, Scolyer RA: Immunohistochemistry is highly sensitive and specific for the detection of V600E BRAF mutation in melanoma. Am J Surg Pathol 2013, 37:61-65.

19. Menzies AM, Haydu LE, Visintin L, Carlino MS, Howle JR, Thompson JF, Kefford RF, Scolyer RA, Long GV: Distinguishing clinicopathologic features of patients with V600E and V600K BRAF-mutant metastatic melanoma. Clin Cancer Res 2012, 18:3242-3249.

20. Skorokhod A, Capper D, von Deimling A, Enk A, Helmbold P: Detection of BRAF V600E mutations in skin metastases of malignant melanoma by monoclonal antibody VE1. J Am Acad Dermatol 2012, 67:488-491.

21. El-Osta H, Falchook G, Tsimberidou A, Hong D, Naing A, Kim K, Wen S, Janku F, Kurzrock R: BRAF mutations in advanced cancers: clinical characteristics and outcomes. PLoS One 2011, 6:e25806.

22. Bettegowda C, Agrawal N, Jiao Y, Wang Y, Wood LD, Rodriguez FJ, Hruban $\mathrm{RH}$, Gallia GL, Binder ZA, Riggins CJ, et al: Exomic Sequencing of Four Rare Central Nervous System Tumor Types. Oncotarget 2013, 4:572-583.

23. Giannini C, Paulus W, Louis DN, Liberski P: Pleomorphic Xanthoastrocytoma in WHO Classification of Tumours of the Central Nervous System (IARC WHO Classification of Tumours). 1st edition. Lyon; 2007:22-24.

24. Giannini C, Scheithauer BW, Burger PC, Brat DJ, Wollan PC, Lach B, O'Neill BP: Pleomorphic xanthoastrocytoma: what do we really know about it? Cancer 1999, 85:2033-2045.

25. Kleinschmidt-Demasters BK, Aisner DL, Birks DK, Foreman NK: Epithelioid GBMs Show a High Percentage of BRAF V600E Mutation. Am J Surg Pathol 2013, 37:685-698.

26. Capper D, Preusser M, Habel A, Sahm F, Ackermann U, Schindler G, Pusch S, Mechtersheimer $G$, Zentgraf $H$, von Deimling A: Assessment of BRAF V600E mutation status by immunohistochemistry with a mutation-specific monoclonal antibody. Acta Neuropathol 2011, 122:11-19.

27. Colomba E, Helias-Rodzewicz Z, Von Deimling A, Marin C, Terrones N, Pechaud D, Surel S, Cote JF, Peschaud F, Capper D, et al: Detection of BRAF p.V600E mutations in melanomas: comparison of four methods argues for sequential use of immunohistochemistry and pyrosequencing. J Mol Diagn 2013, 15:94-100.

28. Feller JK, Yang S, Mahalingam M: Immunohistochemistry with a mutation-specific monoclonal antibody as a screening tool for the BRAFV600E mutational status in primary cutaneous malignant melanoma. Mod Pathol 2013, 26:414-420.

29. Bullock M, O'Neill C, Chou A, Clarkson A, Dodds T, Toon C, Sywak M, Sidhu $\mathrm{SB}$, Delbridge LW, Robinson BG, et al: Utilization of a MAB for BRAF(V600E) detection in papillary thyroid carcinoma. Endocr Relat Cancer 2012, 19:779-784

30. Koperek O, Kornauth C, Capper D, Berghoff AS, Asari R, Niederle B, von Deimling A, Birner P, Preusser M: Immunohistochemical detection of the BRAF V600E-mutated protein in papillary thyroid carcinoma. Am J Surg Pathol 2012, 36:844-850

31. Andrulis M, Penzel R, Weichert W, von Deimling A, Capper D: Application of a BRAF V600E mutation-specific antibody for the diagnosis of hairy cell leukemia. Am J Surg Pathol 2012, 36:1796-1800.

32. Bosmuller $H$, Fischer A, Pham DL, Fehm T, Capper D, von Deimling A, Bonzheim I, Staebler A, Fend F: Detection of the BRAF V600E mutation in serous ovarian tumors: a comparative analysis of immunohistochemistry with a mutation-specific monoclonal antibody and allele-specific PCR. Hum Pathol 2013, 44:329-335.

33. Ilie M, Long E, Hofman V, Dadone B, Marquette CH, Mouroux J, Vignaud JM, Begueret H, Merlio JP, Capper D, et al: Diagnostic value of immunohistochemistry for the detection of the BRAFV600E mutation in primary lung adenocarcinoma Caucasian patients. Ann Oncol 2013, 24:742-748.

34. Capper D, Berghoff AS, Magerle M, Ilhan A, Wohrer A, Hackl M, Pichler J, Pusch S, Meyer J, Habel A, et al: Immunohistochemical testing of BRAF V600E status in 1,120 tumor tissue samples of patients with brain metastases. Acta Neuropathol 2012, 123:223-233.

35. Sperveslage J, Gierke M, Capper D, Honegger J, Sipos B, Beschorner R, Schittenhelm J: VE1 immunohistochemistry in pituitary adenomas is not associated with BRAF V600E mutation. Acta Neuropathol 2013, 125:911-912.

36. Landis JR, Koch GG: The measurement of observer agreement for categorical data. Biometrics 1977, 33:159-174.

37. Fleiss JL, Levin B, Paik MC, Fleiss J: Statistical Methods for Rates \& Proportions Third edn. New York: Wiley-Interscience; 2003.

38. Fleiss $J$, Nee JC, Landis JR: Large sample variance of kappa in the case of different sets of raters. Psychol Bull 1979, 86:974-977.

39. Kendall MG: Rank correlation methods. London: Charles Griffin \& Co. Ltd.; 1955.

doi:10.1186/2051-5960-1-20

Cite this article as: Ida et al:: Immunohistochemistry is highly sensitive and specific for detection of BRAF V600E mutation in pleomorphic xanthoastrocytoma. Acta Neuropathologica Communications 2013 1:20.

\section{Submit your next manuscript to BioMed Central and take full advantage of:}

- Convenient online submission

- Thorough peer review

- No space constraints or color figure charges

- Immediate publication on acceptance

- Inclusion in PubMed, CAS, Scopus and Google Scholar

- Research which is freely available for redistribution

Submit your manuscript at www.biomedcentral.com/submit
C) Biomed Central 